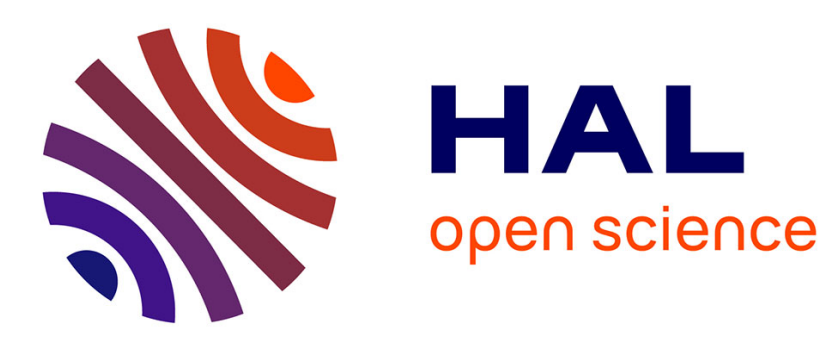

\title{
Robust local stabilization of discrete time-varying delayed state systems under saturating actuators
}

Michelle F.F. Castro, Alexandre Seuret, Valter J.S. Leite, Luis F.P. Silva

\section{To cite this version:}

Michelle F.F. Castro, Alexandre Seuret, Valter J.S. Leite, Luis F.P. Silva. Robust local stabilization of discrete time-varying delayed state systems under saturating actuators. Automatica, 2020, 122, pp.109266. 10.1016/j.automatica.2020.109266 . hal-02995778

\section{HAL Id: hal-02995778 \\ https://hal.science/hal-02995778}

Submitted on 25 Nov 2020

HAL is a multi-disciplinary open access archive for the deposit and dissemination of scientific research documents, whether they are published or not. The documents may come from teaching and research institutions in France or abroad, or from public or private research centers.
L'archive ouverte pluridisciplinaire $\mathbf{H A L}$, est destinée au dépôt et à la diffusion de documents scientifiques de niveau recherche, publiés ou non, émanant des établissements d'enseignement et de recherche français ou étrangers, des laboratoires publics ou privés. 


\title{
Robust local stabilization of discrete time-varying delayed state systems under saturating actuators
}

\author{
Michelle F. F. Castro $^{\text {a }}$, Alexandre Seuret ${ }^{\text {b }}$, Valter J. S. Leite ${ }^{\text {a }}$, Luis F. P. Silva ${ }^{\text {a }}$ \\ ${ }^{a}$ Department of Mechatronics Engineering, CEFET-MG, Campus Divinópolis, 35503-822 Divinópolis/ MG, Brazil \\ ${ }^{\mathrm{b}}$ CNRS, LAAS, 7 avenue du Colonel Roche, 31077 Toulouse, France.
}

\begin{abstract}
This paper deals with discrete time-varying systems with state-delayed and saturating actuators. A robust state feedback control gain is designed through convex methods ensuring the local stability of the time-varying system with interval timevarying state delay. The estimate of the set of admissible initial conditions is characterized by three convex sets allowing less conservative estimates. Through two numerical examples, we compare our approach with others in the literature and illustrate the better behavior of the proposed methods.
\end{abstract}

Key words: Time-varying discrete-time systems, time-varying state-delays, saturating input, robust control.

\section{Introduction}

Processes in the real-world are affected by delays, usually coming from energy, mass, or information transportation. As a consequence, degradation on the performance may occur as well as the stability of the closed-loop system [4]. Additionally, the presence of saturating actuators [19], constraints on the state [18], or switched modes [7] requires the local stability investigation because of some initial sequence of state can lead to different equilibrium points or even to unstable behavior. Thus, it is necessary to estimate a subset of the initial conditions ensuring the respective trajectories to go to the origin asymptotically [19].

It is possible to find several works dealing with the stability of the discrete-time delayed systems in the literature. Recently, a key issue in the research on this field is to obtain less conservative bounds on some integral or summation inequalities, yielding less conservative stability conditions. The reader may refer for instance to $[8,9,14,21]$. While these aspects have now been addressed

\footnotetext{
‡ The paper was partially supported by CAPES and CNPq (311208/2019-3) and has been developed during a stage of the first author on LAAS.

Email addresses: michelleffc@hotmail.com (Michelle F. F. Castro), aseuret@laas.fr (Alexandre Seuret), valter@ieee.org (Valter J. S. Leite), luis@cefetmg.br (Luis F. P. Silva).
}

properly in the literature, the problem of providing nonrestrictive constructive stabilization conditions, i.e., conditions that deliver as an output a state feedback control gain, is still an open issue. Indeed, transforming stability LMI conditions to constructive stabilization conditions is usually made possible at the price of introducing tuning parameters, which imposes conservative restriction as in [5], to cite only one.

To show that this method goes to beyond linear systems with delays, we will also consider additional difficulties in the paper, which consist of having nonlinear dynamics. Indeed, nonlinearities in a system are well known to easily degenerate performance and turn the closed-loop unstable. See [18] for the design of a group of adaptive controllers and a switching law to asymptotically regulate of state-constrained high-order switched nonlinear systems by using multiple-barrier Lyapunov functions. In [16] and [17], a fuzzy-based approach has been used with the generalized sector condition to handle nonlinearities and constraints on the region of model validity. In particular, saturating actuators in discrete-time delayed systems have received attention as one can see in $[22,24,1,15,2,3]$. Due to saturating actuators, the study of the closed-loop behavior and the characterization of the set of initial admissible conditions (usually called domain or region of attraction) require the local stability analysis. Such a characterization is in practice much more difficult to handle for time-delay systems than for delay-free systems. This is because the initial condition 
of a discrete time-delay system is a sequence of vectors specifying the position of the system for negative time instants. Therefore, it is more complicated to efficiently characterize this domain of attraction because one has to constraint the whole sequence of initial conditions, which can be done in several ways. For instance, the region of attraction provided in [22] is defined by imposing that all the components of the initial conditions are uniformly bounded. An alternative characterization was provided in [2], where the initial conditions are constraints to lies into two balls, one for the norm of the sequence of initial conditions, and the other related to the norm of the variation between two consecutive components. Extending this idea, the authors of $[3,15]$ provided a more general characterization through the definition of augmented state space and an associated ellipsoidal set, at the price of a high computational burden. Such an approach was considered first in [6], where the timedelay system is rewritten as a switched delay-free system, and the delay is the switching function. In contrast with other switching approaches, such as that in [7], the one in $[3,6,15]$ does not require dwell-time to achieve the stabilization.

Differently from $[3,15]$, we would like to avoid considering the augmented switched delay-free system approach to keep a low numerical complexity on the proposed conditions. Interestingly, [16] proposed an intermediate characterization, based on useful and simple sets, such as an ellipsoidal one for the current state and a ball concerning both the norm of the initial conditions' sequence and its variations. However, in particular, the LyapunovKrasovskii functionals (LKFs) employed in $[2,16]$ do not consider the coupling between the current state and the previous ones, leading to independent (and conservative) estimates of the region of attraction.

The contributions of the paper are two-fold. After presenting a summation of inequality accounting for systems with time-varying delays similarly as in [14] for the continuous-time case, a method to derive a new constructive stabilization criteria is provided. Second, we provide a new characterization of the admissible set of initial sequences for linear systems subject to time-varying delays and input saturation. It consists in the definition of three sets, allowing a more relaxed characterization of the admissible set of initial conditions than approaches usually encountered in the literature. Finally, two academic examples evaluate our method, where insightful comparisons with recent methods from the literature are established.

Notation: Sets $\mathbb{N}, \mathbb{R}, \mathbb{R}^{n}$, and $\mathbb{R}^{n \times m}$ denote, respectively, the sets of positive integers, real numbers, real $n$ dimensional vectors, real matrices of dimensions $n \times m$, and real square symmetric positive semi-definite matrices of dimensions $n \times n$. For any integers $a \leq b$, notation $\mathcal{I}[a, b]$ stands for $[a, b] \cap \mathbb{N} . P \in \mathbb{S}_{+}^{n}\left(\right.$ or $\left.\mathbb{S}^{n}\right)$ and $P \succ 0(\succeq 0)$ denotes that $P$ is a positive definite (semi- definite) matrix. Matrices $I$ and 0 refers to as the identity and null matrices of appropriate dimensions, respectively. For any matrices $A$ and $B, A^{\top}$ represents the transpose of matrix $A$ and $\operatorname{diag}(A, B)$, the block diagonal matrix $\left[\begin{array}{ll}A & 0 \\ 0 & B\end{array}\right]$. Moreover, if $A$ is a square matrix, $\mathrm{He}(A)$ stands for $A+A^{\top}$. Symbol $\star$ in a square matrix represents the symmetric entry of a symmetric matrix. For any vector (matrix) $G, G_{(\ell)}$ denotes the $\ell^{\text {th }}$ component (line) of $G$, and, to avoid any confusion, $G_{(\ell)}^{\top}$ stands for the transpose of the $\ell^{\text {th }}$-line of $G$. The Euclidean norm is denoted as $|\cdot|$. Set $\mathbb{E}_{\bar{d}}^{n}$ is the set of sequence of $\bar{d}+1$ vectors in $\mathbb{R}^{n}$. More precisely, the elements of $\varphi_{0} \in \mathbb{E}_{\bar{d}}^{n}$ are $\bar{d}+1$ vectors $x_{j} \in \mathbb{R}^{n}$, with $j \in \mathcal{I}[-\bar{d}, 0]$, being denoted as $\varphi_{0}=\left\{x_{-j}\right\}_{j \in \mathcal{I}[0, \bar{d}]}$ and $\varphi_{0}(j)=x_{-j} \cdot \bar{\varphi}_{0} \equiv \varphi_{0} \backslash\left\{x_{0}\right\}$ is obtained from the sequence $\varphi_{0}$ with the element $x_{0}$ removed. The sequence variation is noted by $\Delta \varphi_{k}=\bar{\varphi}_{k+1}-\bar{\varphi}_{k}$, and is composed by $\bar{d}$ elements $\Delta \varphi_{k}=\left\{x_{k+j}-x_{k+j-1}\right\}_{j \in \mathcal{I}[0, \bar{d}-1]}$. $\left\|\varphi_{k}\right\|=\max _{j \in \mathcal{I}[0, \bar{d}]}\left|x_{k-j}\right|$ denotes the norm is associated to $\mathbb{E}_{\bar{d}}^{n}$.

\section{Problem formulation}

Consider the discrete-time system with time-varying state delay and saturating actuator given by

$$
\left\{\begin{array}{l}
x_{k+1}=A x_{k}+A_{d} x_{k-d_{k}}+B \operatorname{sat}\left(u_{k}\right), \quad \forall k \in \mathbb{N}, \\
x_{-j}=\varphi_{0}(j), \quad \forall j \in \mathcal{I}[0, \bar{d}]
\end{array}\right.
$$

where $x_{k}$ and $u_{k}$ are the instantaneous state vector and the control input, respectively. Matrices $A$ and $A_{d} \in$ $\mathbb{R}^{n \times n}$, and $B \in \mathbb{R}^{n \times m}$ belong to a polytope given by the convex combination of $N$ known vertices, such that

$$
\left[\begin{array}{lll}
A & A_{d} & B
\end{array}\right]=\sum_{i=1}^{N} \alpha_{k(i)}\left[\begin{array}{lll}
A_{i} & A_{d i} & B_{i}
\end{array}\right]
$$

where matrices $A_{i}, A_{d i}$, and $B_{i}$ are constant and known, and $\alpha_{k}$ belongs to the unitary simplex

$$
\Gamma=\left\{\alpha_{k} \in \mathbb{R}^{N}: \sum_{i=1}^{N} \alpha_{k(i)}=1, \alpha_{k(i)} \geq 0, i \in \mathcal{I}[1, N]\right\}
$$

The saturation function sat $\left(u_{k}\right)$ is considered componentwise so that

$$
\operatorname{sat}\left(u_{k}\right)_{(\ell)}=\operatorname{sign}\left(u_{k(\ell)}\right) \min \left(\left|u_{k(\ell)}\right|,\left(\bar{u}_{(\ell)}\right)\right),
$$

for $\ell=\mathcal{I}[1, m]$. We recall that notation $u_{k(\ell)}$ and $\bar{u}_{(\ell)}>0$ stand, respectively, for the $\ell^{t h}$ component of vectors $u_{k}$ and $\bar{u} \in \mathbb{R}^{m}$ that contains the maximum magnitudes allowed to each control signal. Sequence $\varphi_{0}$ in $\mathbb{E}_{\bar{d}}^{n}$ denotes 
the initial conditions of the time-delay system. We assume that there exist two nonnegative integers $\underline{d}<\vec{d}$ such that

$$
d_{k} \in \mathcal{I}[\underline{d}, \bar{d}], \quad \forall k \in \mathbb{N} .
$$

The following state-feedback control law is used to stabilize the system (1):

$$
u_{k}=K x_{k},
$$

where $K \in \mathbb{R}^{m \times n}$ is the control gain to be defined.

Because of the saturation, guaranteeing the global stability of the closed-loop system is not possible in general. One has to derive a local stability analysis consisting of the characterization of allowed or admissible sequences of initial conditions, $\varphi_{0}$, which ensure that the closedloop trajectories do not leave the region of attraction, $\mathcal{R}_{\mathcal{A}} \subseteq \mathbb{E}_{\bar{d}}^{n}$. As the computation of the set $\mathcal{R}_{\mathcal{A}}$ is a challenging task, even for low order and delay-free systems (see [19] for more details), one can only compute an estimate of such a region, $\mathcal{R}_{\mathcal{E}} \subseteq \mathcal{R}_{\mathcal{A}}$ and try to make this estimation as large as possible.

There exist many ways to derive estimates of the region of attraction. For instance, in [22], $\mathcal{R}_{\mathcal{E}}$ is characterized as the sequences such that $\left\|\varphi_{0}\right\| \leq r_{1}$, meaning that all elements of sequence are uniformly bounded. A more relaxed characterization was provided in [2] which considers an additional uniform bound $r_{2}$ on the sequence variation, i.e. $\max _{j=1, \ldots, \bar{d}}\left|x_{-j}-x_{-j+1}\right| \leq r_{2}$, respectively. In the present paper, we address the following problem:

Problem 1 Given the state-feedback control gain $K$, verify the local robust stability of the closed-loop system (11). Furthermore, estimate $\mathcal{R}_{\mathcal{E}} \subseteq \mathcal{R}_{\mathcal{A}}$ ensuring that all trajectories emanating from $\varphi_{0} \in \mathcal{R}_{\mathcal{E}}$ converge to the origin without leaving $\mathcal{R}_{\mathcal{E}}$.

Problem 2 Determine the control gain $K$ and an estimate $\mathcal{R}_{\mathcal{E}} \subseteq \mathcal{R}_{\mathcal{A}}$ that ensures the local robust stability of the closed-loop system (1)-(5) for any sequence $\varphi_{0} \in \mathcal{R}_{\mathcal{E}}$.

\section{Preliminary Results}

\subsection{Generalized sector condition [19]}

Let consider the dead-zone function $\phi \in \mathbb{R}^{m}$ defined by

$$
\phi(u)=\operatorname{sat}(u)-u, \quad \forall u \in \mathbb{R}^{m} .
$$

Following [19], a polyhedral set can be defined to handle the saturation (1) with the help of an auxiliary signal $\omega \in \mathbb{R}^{m}$

$$
\mathcal{S}(\omega, \bar{u})=\left\{u \in \mathbb{R}^{m}:\left|(u-\omega)_{(\ell)}\right| \leq \bar{u}_{(\ell)}, \forall \ell \in \mathcal{I}[1, m]\right\}
$$

It is worth noting that introducing $\omega$ relaxes the bounds on $u$, allowing it to go beyond the limits $\pm \bar{u}$.

Lemma 1 For given auxiliary vector $\omega$ and saturation bounds, $\bar{u}$, if vector $u$ belongs to $\mathcal{S}(\omega, \bar{u})$, then the function $\phi(u)$ defined in (6) satisfies the following inequality:

$$
\phi(u)^{\top} \mathcal{T}[\phi(u)+\omega] \leq 0,
$$

for any positive defined diagonal matrix $\mathcal{T} \in \mathbb{S}_{+}^{m}$.

Therefore, Lemma 1 guarantees that having signals $u$ and $\omega$ belonging to $\mathcal{S}(\omega, \bar{u})$ ensures (8). This property will be used in the next section to relax the stabilization conditions.

\subsection{A summation inequality free of slack variables}

In the sequel, we present a lemma that helps us to establish our main result in the next section.

Lemma 2 Let $R \in \mathbb{S}_{+}^{n}$ and $\varphi_{0}$ a sequence in $\mathbb{E}_{\bar{d}}^{n}$. Then, for any integer $c \in \mathcal{I}[\underline{d}, \bar{d}]$, inequality

$$
(\bar{d}-\underline{d}) \sum_{j=-\bar{d}}^{-\underline{d}-1} y_{j}^{\top} R y_{j} \geq \Omega_{c}^{\top} R \Omega_{c}
$$

holds where

$$
\begin{array}{ll}
y_{j}=x_{j}-x_{j-1}, & \forall j \in \mathcal{I}[-\bar{d}+1,0], \\
\Omega_{c}=x_{-\underline{d}}-2 x_{-c}+x_{-\bar{d}}, & \forall c \in \mathcal{I}[\underline{d}, \bar{d}] .
\end{array}
$$

Proof: The proof is first based on the Jensen inequality's application to the left-hand side of (9) after splitting the summation into two parts. More precisely, we have that

$$
\begin{aligned}
\mathcal{J} & :=(\bar{d}-\underline{d})\left[\sum_{j=-c}^{-\underline{d}-1} y_{j}^{\top} R y_{j}+\sum_{j=-\bar{d}}^{-c-1} y_{j}^{\top} R y_{j}\right] \\
& \geq \frac{1}{\alpha} \Omega_{c}^{+\top} R \Omega_{c}^{+}+\frac{1}{1-\alpha} \Omega_{c}^{-\top} R \Omega_{c}^{-}
\end{aligned}
$$

where $\Omega_{c}^{+}=x_{-\underline{d}}-x_{-c}, \Omega_{c}^{-}=x_{-c}-x_{-\bar{d}}$, and $\alpha=\frac{c-\underline{d}}{d-\underline{d}}$. Following the principle of the reciprocally convex combination lemma [11], the previous expression can be rewritten as follows

$$
\mathcal{J} \geq\left[\begin{array}{l}
\Omega_{c}^{+} \\
\Omega_{c}^{-}
\end{array}\right]^{\top}\left(\left[\begin{array}{cc}
\frac{1-\alpha}{\alpha} R & R \\
R & \frac{\alpha}{1-\alpha} R
\end{array}\right]+\left[\begin{array}{cc}
R & -R \\
-R & R
\end{array}\right]\right)\left[\begin{array}{l}
\Omega_{c}^{+} \\
\Omega_{c}^{-}
\end{array}\right]
$$

Let us now note that the first term is positive semidefinite since inequality $\left[\begin{array}{cc}\frac{1-\alpha}{\alpha} R & R \\ R & \frac{\alpha}{1-\alpha} R\end{array}\right] \succeq 0$ is equivalent 
by Schur complement to $\frac{1-\alpha}{\alpha}(R-R)=0$. The result is then derived from

$$
\left[\begin{array}{l}
\Omega_{c}^{+} \\
\Omega_{c}^{-}
\end{array}\right]^{\top}\left[\begin{array}{cc}
R & -R \\
-R & R
\end{array}\right]\left[\begin{array}{l}
\Omega_{c}^{+} \\
\Omega_{c}^{-}
\end{array}\right]=\left(\Omega_{c}^{+}-\Omega_{c}^{-}\right)^{\top} R\left(\Omega_{c}^{+}-\Omega_{c}^{-}\right),
$$

which proofs the lemma.

Remark 1 Note that, taking $c=-\bar{d}$ in (9) yields the usual Jensen inequality for discrete-time delay systems.

Remark 2 The benefits of the inequality in Lemma 2 over the reciprocally convex combination lemma from [11] are a lower number of decision variables and less LMIs to solve since no additional slack variables, and no additional constraints are required. Moreover, it was shown in [12], that the same level of conservatism as in [11] was obtained for varying-delay systems, in the case of Jensenbased stability conditions. A discussion on the conservatism of the previous summation inequality is presented later in this paper concerning more advanced summation inequalities.

\subsection{Stabilization of the closed-loop model}

Because of the saturating actuator in (1) the local stability is required to handle the design of the robust state feedback control gain $K$ in (5). Following the generalized section conditions provided in Lemma 1 from [19], we rewrite system (1) using the dead-zone function as follows

$$
x_{k+1}=A_{c \ell} x_{k}+A_{d} x_{k-d_{k}}+B \phi\left(u_{k}\right),
$$

where $A_{c \ell}=A+B K$ is still an uncertain matrix belonging to the polytope $(2)$, and $\phi$ is the dead-zone function given in (6).

We address the stabilization of system (11) by using the following simple LKF candidate given by

$$
V\left(\varphi_{k}\right)=V_{1}\left(\varphi_{k}\right)+V_{2}\left(\varphi_{k}\right)+V_{3}\left(\varphi_{k}\right)
$$

where

$$
\begin{aligned}
V_{1}\left(\varphi_{k}\right) & =x_{k}^{\top} \bar{P} x_{k}, \\
V_{2}\left(\varphi_{k}\right) & =\sum_{i=k-\underline{d}}^{k-1} x_{i}^{\top} \bar{Q}_{1} x_{i}+\sum_{i=k-\bar{d}}^{k-\underline{d}-1} x_{i}^{\top} \bar{Q}_{2} x_{i}, \\
V_{3}\left(\varphi_{k}\right) & =\underline{d} \sum_{i=1-\underline{d}}^{0} \sum_{j=k+i}^{k} y_{j}^{\top} \bar{Z}_{1} y_{j} \\
& +(\bar{d}-\underline{d}) \sum_{i=1-\bar{d}}^{-\underline{d}} \sum_{j=k+i}^{k} y_{j}^{\top} \bar{Z}_{2} y_{j},
\end{aligned}
$$

where $y_{j}$ is defined in (10), $\varphi_{k} \in \mathbb{E}_{\bar{d}}^{n}$ refers to the functional state of the time-delay system given by $\varphi_{k}(i)=$ $x_{k-j}$ for all $j \in 0, \bar{d}$, and matrices $\bar{P}, \bar{Q}_{i}$, and $\bar{Z}_{i}$, for $i=1,2$, belonging to $\mathbb{S}_{+}^{n}$.

\section{Main results}

\subsection{Constructive stabilization conditions}

In this section, we provide two main results, where one concerns the local stability analysis of the closed-loop system (11), and the other provides a convex control synthesis condition for the local stabilization of (1).

Theorem 1 Consider the uncertain system (1) under the control law (5) with a given control gain $K$. Suppose that there exist matrices $W, Q_{1}, Q_{2}, Z_{1}$, and $Z_{2}$ in $\mathbb{S}_{+}^{n}$, $G \in \mathbb{R}^{m \times 4 n}$, a positive diagonal matrix $\mathcal{T} \in \mathbb{S}_{+}^{m}$, and a scalar $\epsilon \in[0,2]$ that satisfy the following LMIs

$$
\begin{aligned}
& {\left[\begin{array}{cc}
\Phi_{1 i} & \Phi_{2 i} \\
\star & \Phi_{3}
\end{array}\right] \prec 0, \forall i \in \mathcal{I}[1, N],} \\
& {\left[\begin{array}{cc}
{\left[\begin{array}{cc}
W & 0 \\
\star & \mathcal{Q}_{1}
\end{array}\right]} & \left.\left(\left[\begin{array}{c}
K W \\
0
\end{array}\right]-G\right)_{(\ell)}^{\top}\right] \\
u_{0(\ell)}^{2}
\end{array}\right] \succeq 0, \forall \ell \in \mathcal{I}[1, m],}
\end{aligned}
$$

where

$$
\begin{aligned}
\Phi_{1 i}= & \operatorname{He}\left(F_{1}^{\top} \Phi_{2 i}^{\top}-F_{5}^{\top} G \Xi\right)+\mathcal{Q}_{2}-2 F_{5}^{\top} \mathcal{T} F_{5} \\
& -\Pi^{\top}\left[\begin{array}{cc}
Z_{1} & 0 \\
\star & Z_{2}
\end{array}\right] \Pi, \\
\Phi_{2 i}= & {\left[A_{i} W+B_{i} K W-W 0 A_{d i} W \quad 0 \quad B_{i} \mathcal{T}\right]^{\top}, } \\
\Phi_{3}= & -\left(2 \epsilon-\epsilon^{2}\right) W+\epsilon^{2}\left(\underline{d}^{2} Z_{1}+(\bar{d}-\underline{d})^{2} Z_{2}\right), \\
\mathcal{Q}_{1}= & \operatorname{diag}\left(Q_{1}, Q_{2}, Q_{2}\right), \\
\mathcal{Q}_{2}= & \operatorname{diag}\left(Q_{1},-Q_{1}+Q_{2}, 0,-Q_{2}, 0\right),
\end{aligned}
$$

with the following matrices

$$
\begin{aligned}
& \Pi=\left[\begin{array}{ccccc}
I & -I & 0 & 0 & 0 \\
0 & I & -2 I & I & 0
\end{array}\right], \quad F_{1}=\left[\begin{array}{lllll}
I & 0 & 0 & 0 & 0
\end{array}\right] \\
& \Xi=\left[\begin{array}{lllll}
I & 0 & 0 & 0 & 0 \\
0 & I & 0 & 0 & 0 \\
0 & 0 & I & 0 & 0 \\
0 & 0 & 0 & I & 0
\end{array}\right], \quad F_{5}=\left[\begin{array}{lllll}
0 & 0 & 0 & 0 & I
\end{array}\right] \text {. }
\end{aligned}
$$

Then the closed-loop system (11) is locally asymptotically stable for any sequence of initial conditions $\varphi_{0}$ verifying

$$
\varphi_{0} \in \mathcal{C}_{v}:=\left\{\varphi_{0} \in \mathbb{E}_{\bar{d}}^{n} \quad \text { s.t. } \quad V\left(\varphi_{0}\right)<1\right\},
$$

where $V$ is the functional in (12)-(15) with, for $i=1,2$

$$
\bar{P}=W^{-1}, \bar{Q}_{i}=W^{-1} Q_{i} W^{-1}, \bar{Z}_{i}=W^{-1} Z_{i} W^{-1}
$$


Proof : Consider the LKF candidate $V\left(\varphi_{k}\right)$ given in (12)-(15) with the parameters provided in (20). Clearly, since matrices $P, Q_{i}$, and $Z_{i}$ are assumed to be symmetric positive definite, the functional is also positive definite. Let us compute the increment of the functional, $\Delta V\left(\varphi_{k}\right):=V\left(\varphi_{k+1}\right)-V\left(\varphi_{k}\right)$. Following the usual computational methods, the increment of the functional can be expressed as follows

$$
\begin{aligned}
\Delta V\left(\phi_{k}\right)= & x_{k+1}^{\top} W^{-1} W W^{-1} x_{k+1} \\
& +y_{k+1}^{\top} W^{-1}\left(\underline{d}^{2} Z_{1}+(\bar{d}-\underline{d})^{2} Z_{2}\right) W^{-1} y_{k+1} \\
& -x_{k}^{\top} W^{-1}\left(W+Q_{1}\right) W^{-1} x_{k} \\
& -x_{k-\underline{d}}^{\top} W^{-1}\left(Q_{1}-Q_{2}\right) W^{-1} x_{k-\underline{d}} \\
& -x_{k-\bar{d}}^{\top} W^{-1} Q_{2} W^{-1} x_{k-\bar{d}} \\
& -\underline{d} \sum_{i=k-\underline{d}+1}^{k} y_{i}^{\top} W^{-1} Z_{1} W^{-1} y_{i} \\
& -(\bar{d}-\underline{d}) \sum_{i=k-\bar{d}+1}^{k} y_{i}^{\top} W^{-1} Z_{2} W^{-1} y_{i} .
\end{aligned}
$$

The next steps consist in replacing $x_{k+1}$ by $y_{k+1}-x_{k}$ in the first term of $\Delta V$, and in applying Lemma 2 to the last two negative terms of the previous expression. Then, by introducing the augmented vector $\xi_{k}$ defined by

$$
\xi_{k}=\left[\begin{array}{c}
\rho_{k} \\
\mathcal{T}^{-1} \psi_{k}
\end{array}\right], \quad \rho_{k}=\left[\begin{array}{c}
W^{-1} x_{k} \\
W^{-1} x_{k-\underline{d}} \\
W^{-1} x_{k-d_{k}} \\
W^{-1} x_{k-\bar{d}}
\end{array}\right],
$$

several manipulations allow us to write the following upper bound:

$$
\Delta V\left(\varphi_{k}\right) \leq \xi_{k}^{\top} \hat{\Phi}_{1} \xi_{k}+\operatorname{He}\left(\phi_{k}^{\top} \mathcal{T}^{-1}\left(\phi_{k}+G \Xi \xi_{k}\right)\right)
$$

where we have used $y_{k+1}=\sum_{i=1}^{N} \alpha_{k(i)} \Phi_{2 i}^{\top} \xi_{k}$ and with

$$
\begin{aligned}
& \hat{\Phi}_{1}=\sum_{i=1}^{N} \alpha_{k(i)} \Phi_{1 i}+\left(\sum_{i=1}^{N} \alpha_{k(i)} \Phi_{2 i}\right)^{\top} \bar{Y}^{-1}\left(\sum_{i=1}^{N} \alpha_{k(i)} \Phi_{2 i}\right) \\
& \bar{Y}=W\left(W+\underline{d}^{2} Z_{1}+(\bar{d}-\underline{d})^{2} Z_{2}\right)^{-1} W
\end{aligned}
$$

Then, the Schur complement ensures that matrix $\hat{\Phi}_{1}$ is negative definite if and only if we have

$$
\left[\begin{array}{cc}
\sum_{i=1}^{N} \alpha_{k(i)} \Phi_{1} & \sum_{i=1}^{N} \alpha_{k(i)} \Phi_{2 i} \\
\star & -\bar{Y}
\end{array}\right]=\sum_{i=1}^{N} \alpha_{k(i)}\left[\begin{array}{cc}
\Phi_{1 i} & \Phi_{2 i} \\
\star & -\bar{Y}
\end{array}\right] \prec 0
$$

Moreover, since $(W-\epsilon Y) Y^{-1}(W-\epsilon Y) \geq 0$ for any matrix $Y$ and scalar $\epsilon$, selecting $Y=W+\underline{d}^{2} Z_{1}+(\bar{d}-$ $\underline{d})^{2} Z_{2}$, we get that $\bar{Y}$ is greater than $\Phi_{3}$, given in (18), which is ensured if conditions (16) are verified.

Therefore, local stability of the closed-loop system is guaranteed if the term $\phi_{k}^{\top} \mathcal{T}^{-1}\left(\phi_{k}+G \Xi \xi_{k}\right)$ is also negative. To do so, we use the generalized sector condition provided in Lemma 1, where we have implicitly introduced the auxiliary vector $\omega_{k}=G \Xi \xi_{k}=G \rho_{k}$. Let us first apply the Schur complement to the last line and column of (17), for a given $\ell$ and then multiply from the right side by $\rho_{k}$ and its transpose from the left side, leading to:

$$
\begin{aligned}
0< & \rho_{k}^{\top}\left\{\left[\begin{array}{cc}
P & 0 \\
\star & \mathcal{Q}_{1}
\end{array}\right]-\left(\left[\begin{array}{c}
K W \\
0
\end{array}\right]-G\right)_{(\ell)}^{\top} \bar{u}_{(\ell)}^{-2}\right. \\
& \left.\times\left(\left[\begin{array}{c}
K W \\
0
\end{array}\right]-G\right)_{(\ell)}\right\} \rho_{k} \\
= & x_{k}^{\top} \bar{P}_{k}+x_{k-{ }_{d}}^{\top} \bar{Q}_{1} x_{k-\underline{d}}+x_{k-d_{k}}^{\top} \bar{Q}_{2} x_{k-d_{k}} \\
& +x_{k-\bar{d}}^{\top} \bar{Q}_{2} x_{k-\bar{d}}-\frac{\left(K x_{k}-\omega_{k}\right)_{(\ell)}^{\top}\left(K x_{k}-\omega_{k}\right)_{(\ell)}}{\bar{u}_{(\ell)}^{2}} \\
\leq & V\left(\varphi_{0}\right)-\bar{u}_{(\ell)}^{-2}\left|\left(u_{k}-\omega_{k}\right)_{(\ell)}\right|^{2},
\end{aligned}
$$

for $\varphi_{0}$ verifying (19). Therefore, with $\varphi_{0} \in \mathcal{C}_{v}$ we ensure that $u_{k}$ belongs to $\mathcal{S}\left(\omega_{k}, \bar{u}\right)$ so that the generalized sector condition in Lemma 1 holds. Hence in this situation, one can guarantee that the increment of the functional is negative defined for any $\varphi_{0} \in \mathcal{C}_{v}$. Since a level surface of the LKF defines the set $\mathcal{C}_{v}$, the solutions cannot leave this region, which concludes the proof.

Remark 3 Observe that the conditions proposed in Theorem 1 with $\epsilon=1$ recovers the particular case where the inequality $(W-Y)^{\top} Y^{-1}(W-Y) \geq 0$ is used (see, for instance, [16]). Therefore, the parameter $\epsilon$ delivers an extra degree of liberty to solve the analysis conditions.

A relevant comment is related to the application of the generalized sector conditions. In the literature of timedelay systems, the auxiliary vector $\omega$ is usually selected as $\hat{G} x_{k}$, with $\hat{G} \in \mathbb{R}^{m \times n}$, while here we were able to also include more information about the functional state of the delay system by including in this auxiliary vector $x_{k-\underline{d}}, x_{k-d_{k}}$ and $x_{k-\bar{d}}$. This modification is made possible through the introduction of the matrix $\mathcal{Q}_{1}$ in condition (17).

Replacing variable product $K W$ by $L$ in the LMI conditions (16)-(18) yields to an equivalent one, allowing the local stabilizing controller design. Therefore, we formalize the design conditions based on the Theorem 1 as follows. 
Theorem 2 Consider the uncertain system (1). Assume that there exist matrices $W, Q_{1}, Q_{2}, Z_{1}$, and $Z_{2}$ in $\mathbb{S}_{+}^{n}$, matrices $L \in \mathbb{R}^{m \times n}, G \in \mathbb{R}^{m \times 4 n}$, and a positive diagonal matrix $\mathcal{T} \in \mathbb{S}_{+}^{m}$, and a scalar $\epsilon \in[0,2]$ that satisfy the LMIs (16) and (17) replacing therein $K W$ by $L$. Then, the control law (5) with static feedback gain

$$
K=L W^{-1},
$$

ensures the local robust local stability of the closed-loop system system (1)-(5) for any sequence of initial conditions $\varphi_{0} \in \mathcal{C}_{v}$ given in (19), which is defined by the $L K F$ given in (12) with (20).

\subsection{Geometrical characterization of $\mathcal{R}_{\mathcal{E}}$}

Theorems 1 and 2 provide constructive conditions to ensure that the closed-loop system (11) with the static feedback control laws (5) or (22) is locally asymptotically stable. They also provide an estimate $\mathcal{R}_{\mathcal{E}}=\mathcal{C}_{v}$ of the region of attraction. A main issue is to understand how to optimize the parameters of the LKF to enlarge the $\mathcal{C}_{v} \subseteq \mathbb{E}_{\bar{d}}^{n}$. Therefore, the initial condition $\varphi_{0}$ is a function, which has $n(\bar{d}+1)$ degrees of freedom. Inspired by the approach in [16] and [17], we propose a characterization of the estimate of the region of attraction taking into account the couplings between $x_{0}$ and $x_{-1}$, namely $y_{0}$ calculated by (10). Here, the admissible sequences of initial conditions $\varphi_{0}$ are such that:

$$
\varphi_{0}=\left\{\varphi \in \mathbb{E}_{\bar{d}}^{n}: x_{0} \in \mathcal{C}, y_{0} \in \mathcal{V}, \bar{\varphi}_{0} \in \mathcal{B}\left(r_{1}, r_{2}\right)\right\}
$$

where $\mathcal{C}$ and $\mathcal{V}$ are ellipsoidal sets confining the admissible values of $x_{0}$ and $y_{0}$, respectively, and the delayed states, $\bar{\varphi}_{0}$, belong to $\mathcal{B}\left(r_{1}, r_{2}\right)$, thus ensuring $\left\|\bar{\varphi}_{0}\right\| \leq r_{1}$ and $\left\|\Delta \bar{\varphi}_{0}\right\| \leq r_{2}$. Therefore, sets $\mathcal{C}, \mathcal{V}$, and $\mathcal{B}\left(r_{1}, r_{2}\right)$ jointly yield a possible geometrical characterization of $\mathcal{R}_{\mathcal{E}}$. The following lemma is used to define the sets $\mathcal{C}$, $\mathcal{V}$ and $\mathcal{B}\left(r_{1}, r_{2}\right)$ mentioned in (23) which jointly characterize the allowed initial conditions for the closed-loop system (11).

Lemma 3 If (12) is an LKF ensuring the local robust stability of (11), then the initial sequences $\varphi_{0}$ verifying (23) with

$$
\begin{aligned}
\mathcal{C} & =\left\{x_{0} \in \mathbb{R}^{n}: V_{1}\left(x_{0}\right) \leq 1-\gamma\right\} \\
\mathcal{V} & =\left\{y_{0} \in \mathbb{R}^{n}: y_{0}^{\top} J y_{0}<1\right\} \\
\mathcal{B}\left(r_{1}, r_{2}\right) & =\left\{\bar{\varphi}_{0} \in \mathbb{E}_{\bar{d}-1}^{n}:\left\|\bar{\varphi}_{0}\right\| \leq r_{1},\left\|\Delta \bar{\varphi}_{0}\right\| \leq r_{2}\right\} \\
\gamma & =\rho_{1}\left\|\bar{\varphi}_{0}\right\|^{2}+\rho_{2}\left\|\Delta \bar{\varphi}_{0}\right\|^{2}+y_{0}^{\top} J y_{0},
\end{aligned}
$$

and $r_{1}$ and $r_{2}$ chosen such that

$$
\begin{aligned}
0 & \leq \rho_{1} r_{1}^{2}+\rho_{2} r_{2}^{2}+y_{0}^{\top} J y_{0}<1, \text { with } \\
J & =\underline{d}^{2} Z_{1}+(\bar{d}-\underline{d})^{2} Z_{2},
\end{aligned}
$$

$$
\begin{aligned}
& \rho_{1}=\underline{d} \eta_{1}+(\bar{d}-\underline{d}) \eta_{2}, \text { and } \\
& \rho_{2}=0.5\left(\underline{d^{2}}(\underline{d}-1) \eta_{3}+(\bar{d}-\underline{d})^{2}(\bar{d}+\underline{d}-1) \eta_{4}\right),
\end{aligned}
$$

where $\eta_{1}=\lambda_{\max }\left(Q_{1}\right), \eta_{2}=\lambda_{\max }\left(Q_{2}\right), \eta_{3}=\lambda_{\max }\left(Z_{1}\right)$, $\eta_{4}=\lambda_{\max }\left(Z_{2}\right)$, are such that the respective trajectories do not leave the estimate region of attraction and go asymptotically to the origin.

Proof : By hypothesis, (12) is an LKF for the closedloop system (11). Consequently, we can define a level set given by $\mathcal{C} \equiv \mathcal{L}_{V_{1}}(1-\gamma)=\left\{x_{k} \in \mathbb{R}^{n}: x_{k}^{\prime} P x_{k} \leq 1-\gamma\right\}$, with $\gamma$ given in (27). By using $V_{2}\left(\varphi_{0}\right)$ and $V_{3}\left(\varphi_{0}\right)$ given in (14)-(15), we get

$$
\begin{aligned}
& V_{2}\left(\varphi_{0}\right)+V_{3}\left(\varphi_{0}\right) \\
& \quad \leq \underline{d} \lambda_{\max }\left(Q_{1}\right)\left\|\bar{\varphi}_{0}\right\|^{2}+(\bar{d}-\underline{d}) \lambda_{\max }\left(Q_{2}\right)\left\|\bar{\varphi}_{0}\right\|^{2} \\
& \quad+0.5 \underline{d}^{2}(\underline{d}-1) \lambda_{\max }\left(Z_{1}\right)\left\|\Delta \bar{\varphi}_{0}\right\|^{2} \\
& \quad+0.5(\bar{d}-\underline{d})^{2}(\bar{d}+\underline{d}-1) \lambda_{\max }\left(Z_{2}\right)\left\|\Delta \bar{\varphi}_{0}\right\|^{2}+y_{0}^{\top} J y_{0} \\
& \quad=\rho_{1}\left\|\bar{\varphi}_{0}\right\|_{\bar{d}}^{2}+\rho_{2}\left\|\Delta \bar{\varphi}_{0}\right\|_{\bar{d}}^{2}+y_{0}^{\top} J y_{0},
\end{aligned}
$$

with $J, \rho_{1}$, and $\rho_{2}$ given by (29)-(31). To ensure $0 \leq \gamma<1$, it is required that (28) be satisfied.

Observe that, the set $\mathcal{V}$ in Lemma 3 handles the transition between the current state and the previous one, which allows to get a less conservative estimate for the ball $\mathcal{B}\left(r_{1}, r_{2}\right)$. Therefore, for each choice of $y_{0}$ verifying (25) we get an estimate for $\mathcal{C}$ and $\mathcal{B}\left(r_{1}, r_{2}\right)$. If we are interested in the largest admissible $y_{0}$, then we need to estimate it through set $\mathcal{V}$. In this case, we get $\left|y_{0}\right| \leq 1 / \sqrt{\rho_{3}}$ with $\rho_{3}=\underline{d}^{2} \eta_{3}+(\bar{d}-\underline{d})^{2} \eta_{4}$. Moreover, the region of attraction can be casted in a ball belonging to $\mathbb{R}^{n}$ with radius $r$ (see [20], [10], [2]) by taking $r_{1}=r$ and $r_{2}=2 r$, and from $(24)$, we have $V_{1}\left(x_{0}\right) \leq 1-\gamma$, which yields to $r \leq\left(\lambda_{\max }(P)+\rho_{1}+4\left(\rho_{2}+\rho_{3}\right)\right)^{-1 / 2}$.

Observe that, theorems 1 and 2 encompass the case of uncertain and time-invariant delay, i.e. $\underline{d}=\bar{d}$. In such a case, the expressions in (30) and (31) are rewritten as $\rho_{1}=\underline{d} \eta_{1}$ and $\rho_{2}=\underline{d}^{2}(1+\underline{d}) \eta_{3} / 2$, respectively.

\subsection{Optimization procedure}

An optimization procedure can be associated to Theorems 1 and 2, which only treat a feasibility problem, to maximize the size of the sets $\mathcal{C}$ and $\mathcal{B}\left(r_{1}, r_{2}\right)$. To this end, we propose the following optimization procedure, where $\beta_{j} \geq 0$ are weights, $H_{j} \in \mathbb{R}^{n \times n}$, and $X_{j}$ corresponds to 
the $j^{\text {th }}$ matrix in the list $\left\{W, Q_{1}, Q_{2}, Z_{1}, Z_{2}\right\}$ :

$\mathrm{J}=\left\{\begin{array}{c}\operatorname{trace}\left(\sum_{j=1}^{5} \beta_{j} H_{j}\right) \\ \text { s.t. }(16),(17), \text { and }\left[\begin{array}{cc}H_{j} & I \\ I & 2 W-X_{j}\end{array}\right] \succeq 0 .\end{array}\right.$

Observe that, if (33) is feasible, then from Schur's complement, we get $H_{j} \succeq\left(2 W-X_{j}\right)^{-1} \succeq W^{-1} X_{j} W^{-1}$. Because of $\bar{X}_{j}=W^{-1} X_{j} W^{-1}$, with $\bar{X}_{j}$ the $j^{t h}$ matrix in the list $\left\{\bar{P}, \bar{Q}_{1}, \bar{Q}_{2}, \bar{Z}_{1}, \bar{Z}_{2}\right\}$, we have $H_{j} \succeq \bar{X}_{j}$ and thus the minimization of $\operatorname{trace}\left(H_{j}\right)$ leads to the minimization of trace $\left(\bar{X}_{j}\right)$. Additionally, $\beta_{j}$ can be used to ponder the effects of each matrix of the LKF candidate.

Remark 4 In this paper, the stabilization conditions results from the application of Lemma 2. This lemma can be seen as a conservative tools since many summation inequalities have been proposed in the recent literature of discrete-time delay systems [13,21,23]. Using these new results would eventually lead to better numerical results. However this would distract the reader about the main message of the paper, which is the introduction of a new method for the estimation of the region of attraction for saturated systems. These improvements are kept for future works.

\section{$5 \quad$ Numerical Examples}

The examples were programmed in the Matlab using YALMIP and the solver lmilab.

Example 1: Consider the discrete-time system (1) with known matrices given by

$$
A=\left[\begin{array}{cc}
1.1 & 0.15 \\
0.03 & 0.8
\end{array}\right], A_{d}=\left[\begin{array}{cc}
0 & -0.1 \\
0 & 0
\end{array}\right], B=\left[\begin{array}{c}
1 \\
0.1
\end{array}\right],
$$

constant delay $\underline{d}=\bar{d}=5$, and $\bar{u}=15$. The objective here is to design the saturating control law (5) such that the closed-loop system has the largest set of initial conditions. By using the optimization procedure $\mathrm{J}$, with $\beta_{2}=1$ and $\beta_{1}=\beta_{3}=\beta_{4}=\beta_{5}=0$, and $\epsilon=$ 1.9052 we get $\rho_{1}=5.8972 \times 10^{-5}, \rho_{2}=3.0851 \times 10^{-7}$, $\rho_{3}=1.5426 \times 10^{-7}$. As we argued before, Theorem 1 can be used to estimate the set of initial conditions as a ball. In this case, we get $r=74.3493$, which is greater than those computed in [2] $(r=72.596)$, [24] $(r=73.693),[22]\left(r=63.029\right.$, with $E=0, \underline{\alpha}_{(1)}=0.5$, $\sigma_{1}=0.15$ and $\left.\sigma_{2}=0.14\right)$, and [1] $(r=68.491$, with $\varepsilon_{1}=2$ and $\epsilon=2 \times 10^{9}$ ). Therefore, this illustrates that our approach leads to a larger ball of initial conditions. However, our approach allows us to propose more general sets than the ones compared here. For instance, if we choose $r_{1}=74$ and $r_{2}=0$, we can pick as a sequence of initial conditions

$$
\varphi_{0}=\left\{\left[\begin{array}{c}
-68.3 \\
68.4
\end{array}\right],\left[\begin{array}{l}
37 \\
64
\end{array}\right],\left[\begin{array}{l}
37 \\
64
\end{array}\right],\left[\begin{array}{l}
37 \\
64
\end{array}\right],\left[\begin{array}{l}
37 \\
64
\end{array}\right],\left[\begin{array}{l}
37 \\
64
\end{array}\right]\right\}
$$

which has a norm $31.1 \%$ greater than the best estimate among $[1,2,24,22]$ with $\left\|\varphi_{0}\right\|=96.6615$. Therefore, our approach allows the designer to handle sequences with norm much greater than those found in the mentioned works.

Example 2: Consider the time-varying system (1) with two vertices given by

$$
\begin{gathered}
A_{1}=\left[\begin{array}{ll}
0.38 & 0.20 \\
0.09 & 1.00
\end{array}\right], A_{d 1}=\left[\begin{array}{cc}
0.01 & -0.03 \\
0.02 & 0
\end{array}\right], B_{1}=\left[\begin{array}{l}
1.98 \\
0.99
\end{array}\right], \\
A_{2}=\left[\begin{array}{ll}
0.42 & 0.20 \\
0.11 & 1.00
\end{array}\right], A_{d 2}=\left[\begin{array}{ll}
0.05 & 0.09 \\
0.04 & 0.06
\end{array}\right], B_{2}=\left[\begin{array}{l}
2.02 \\
1.01
\end{array}\right] .
\end{gathered}
$$

The delay verifies $d_{k} \in \mathcal{I}[2,4]$, and saturating control signal with $\bar{u}=10$. As done in the Example 1 , we firstly use the optimization procedure $\mathrm{J}$ with $\beta_{1}=\beta_{2}=\beta_{3}=0$, $\beta_{4}=\beta_{5}=1$ and $\epsilon=1.678$ to compute the bigger ball in $\mathbb{R}^{2}\left(r_{1}=r\right.$ and $\left.r_{2}=2 r\right)$ for initial conditions $\varphi_{0}$. In this case, we found $r=55.2278$ which yields the ball $\mathcal{B}_{1}$ and the ellipsoid $\mathcal{C}_{1}$ shown in Figure 1 (dashed black line). If the delayed states are assumed to be the half of this

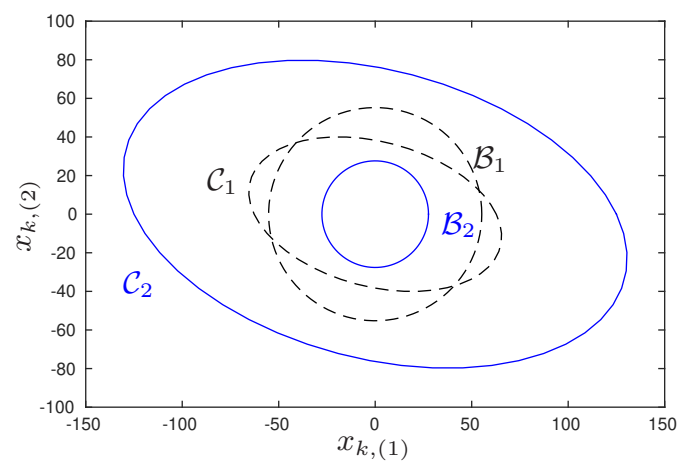

Fig. 1. Region of attraction.

value, i.e., $r=27.6139$, then we get the ball $\mathcal{B}_{2}$ and the ellipsoid $\mathcal{C}_{2}$ shown in Figure 1 (solid blue line). In this last case the sequences $\varphi_{0}$ can have norm as great as 139.2372. Also note that, as the radius $r_{1}$ (with $r_{2}=2 r_{1}$ ) shrinks, the size of the region $\mathcal{C}$ grows up.

We have investigated this effect by taking equally spaced values of $5.52278 \leq r \leq 55.2278$ : for each value of $r$, with 
$r_{1}=r$ and $r_{2}=2 r$, we take vectors $x_{-1} \in \mathbb{R}^{2}$ in the border of the set $\mathcal{B}(r, 2 r)$ (thus, $x_{-1}$ with modulus equal to $r$ and angle $\theta$ with the horizontal axis swiped over a half cycle). For each pair $(r, \theta)$ we have calculated the area of the respective set $\mathcal{C}: \mathcal{S}_{\mathcal{C}}=\pi(1-\gamma) / \sqrt{\operatorname{det} P}$ and $\gamma$ given by (27). The achieved areas are shown in Figure 2 where it is clear the area's size improvement of the set

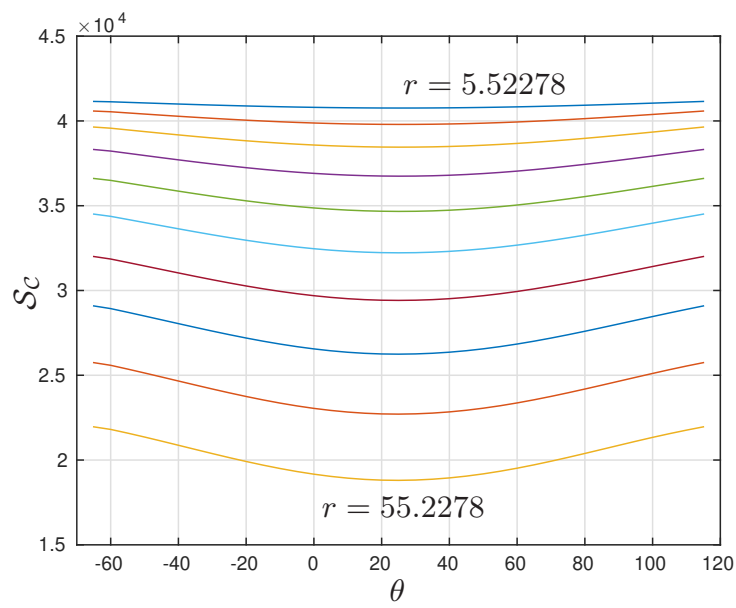

Fig. 2. Area of $\mathcal{C}, \mathcal{S}_{\mathcal{C}}$, for $5.52278 \leq r \leq 55.2278$ and $x_{-1}$ taken on the border of $\mathcal{B}(r, 2 r)$.

$\mathcal{C}$ (associated with $x_{0}$ ) as the norm of the delayed states is reduced. Thus, this illustrates how our proposal of the region of attraction's estimate can yield less conservative results concerning other conditions found in the literature.

\section{Conclusion}

We have provided a new convex delay-dependent condition robust local controller design for a class of discrete time-varying delayed state systems under saturating actuators. We also gave less conservative conditions to robust local stabilize the considered class of systems from new bounds on sum inequalities and a new set characterization of initial admissible sequences. We illustrate how the proposed conditions can be used to get larger sequences of initial conditions through numerical examples.

\section{References}

[1] Y. Chen, S. Fei, and K. Zhang. Stabilisation for switched linear systems with time-varying delay and input saturation. International Journal of Systems Science, 45(3):532-546, 2014.

[2] Y. Chen, Z. Wang, S. Fei, and Q-L. Han. Regional stabilization for discrete time-delay systems with actuator saturations via a delay-dependent polytopic approach. IEEE Transactions on Automatic Control, 64(3):1257-1264, 2019.
[3] C. De Souza, V. J. S. Leite, L. F. P. Silva, and E. B. Castelan. ISS robust stabilization of state-delayed discretetime systems with bounded delay variation and saturating actuators. IEEE Transactions on Automatic Control, 64:3913 - 3919, 2019.

[4] E. Fridman. Introduction to Time-Delay Systems. Systems \& Control: Foundations \& Applications. Birkhäuser, 2014.

[5] Y. He, M. Wu, G.-P. Liu, and J.-H. She. Output feedback stabilization for a discrete-time system with a time-varying dela. IEEE Transactions on Automatic Control, 53(10):2372 - 2377, 2008.

[6] L. Hetel, J. Daafouz, and C. Iung. Equivalence between the Lyapunov-Krasovskii functionals approach for discrete delay systems and that of the stability conditions for switched systems. Nonlinear Analysis: Hybrid Systems, 2(3):697 - 705, 2008.

[7] J. Li, R. Ma, G. M. Dimirovski, and J. Fu. Dwell-timebased stabilization of switched linear singular systems with all unstable-mode subsystems. Journal of the Franklin Institute, 354(7):2712 - 2724, 2017.

[8] X. Liu, F. Wang, and M. Tang. Auxiliary function-based summation inequalities and their applications to discretetime systems. Automatica, 78:211-215, 2017.

[9] P. Nam, P. Pathirana, and H. Trinh. Discrete wirtinger-based inequality and its application. International Journal of the Franklin Institute, 352:1893-1905, 2015.

[10] V. C. Pal and R. Negi. Delay-dependent stability criterion for uncertain discrete time systems in presence of actuator saturation. Transactions of the Institute of Measurement and Control, 40(6):1873-1891, 2018.

[11] P. G. Park, J. W. Ko, and C. Jeong. Reciprocally convex approach to stability of systems with time-varying delays. Automatica, 47:235 - 238, 2011.

[12] A. Seuret and F. Gouaisbaut. Integral inequality for timevarying delay systems. European Control Conference France, 2014.

[13] A. Seuret, F. Gouaisbaut, and E. Fridman. Stability of discrete-time systems with time-varying delays via a novel summation inequality. IEEE Transactions on Automatic Control, 60(10):2740-2745, 2015.

[14] A. Seuret, F. Gouaisbaut, and K. Liu. Discretized Jensen inequality: an alternative vision of the reciprocally convex combination lemma. IFAC Papers OnLine, 2016.

[15] J. V. V. Silva, L. F. P. Silva, I. Rubio Scola, and V. J. S. Leite. Robust local stabilization of discrete-time systems with timevarying state delay and saturating actuators. Mathematical Problems in Engineering, 2018:1-9, 2018. Article ID 5013056.

[16] L. F. P. Silva, V. J. S. Leite, E. B. Castelan, and G. Feng. Delay dependent local stabilization conditions for time-delay nonlinear discrete-time systems using takagi-sugeno models. International Journal of Control, Automation and Systems, 16:1435-1447, 2018.

[17] L. F. P. Silva, V. J. S. Leite, E. B. Castelan, M. Klug, and K. Guelton. Local stabilization of nonlinear discrete-time systems with time-varying delay in the states and saturating actuators. Information Sciences, 518:272-285, May 2020.

[18] Y. Sun, J. Zhao, and G. M. Dimirovski. Adaptive control for a class of state-constrained high-order switched nonlinear systems with unstable subsystems. Nonlinear Analysis: Hybrid Systems, 32:91-105, 2019.

[19] S. Tarbouriech, G. Garcia, J. M. Gomes da Silva Jr., and I. Queinnec. Stability and stabilization of linear systems with saturating actuators. Springer Science \& Business Media, 2011. 
[20] Y. Wei, W. X. Zheng, and S. Xu. Robust output feedback control of uncertain time-delay systems with actuator saturation and disturbances. Journal of the Franklin Institute, 352(5):2229-2248, 2015.

[21] S. Xiao, L. Xu, H.-B. Zeng, and K. L. Teo. Improved stability criteria for discrete- time delay systems via novel summation inequalities. International Journal of Control, Automation and Systems, 16:1592-1602, 2018.

[22] S. Xu, G. Feng, Y. Zou, and J. Huang. Robust controller design of uncertain discrete time-delay systems with input saturation and disturbances. IEEE Transactions on Automatic Control, 57(10):2604-2609, 2012.

[23] C.-K. Zhang, Y. He, L. Jiang, M. Wu, and H.-B. Zeng. Summation inequalities to bounded real lemmas of discretetime systems with time-varying delay. IEEE Transactions on Automatic Control, 62(5):2582-2588, 2017.

[24] X. Zhang, J. Zhao, and G. M. Dimirovski. $l_{2}$-gain analysis and control synthesis of uncertain discrete-time switched linear systems with time delay and actuator saturation. International Journal of Control, 84(10):1746-1758, 2011. 